\title{
NHÂN GIỐNG LOÀI RÂU HÙM (TACCA CHANTRIERI ANDRE) BĂNG PHƯƠNG PHÁP NHÂN GIỐNG SINH DƯỠNG Ở TRƯỜNG ĐẠI HỌC TÂN TRÀO, TUYÊN QUANG
}

\author{
Nguyễn Thị Hải ${ }^{a}$, Trần Thị Thanh Vân ${ }^{a}$, Chu Quỳnh Mai ${ }^{a^{*}}$ \\ ${ }^{a}$ Truò̀ng Đại học Tân Trào \\ *Email: maiquynhtytq82@gmail.com
}

Thông tin bài viết

Ngày nhận bài:

20/4/2020

Ngày duyệt đăng:

$12 / 8 / 2020$

Tù khóa:

Giâm hom, Râu hùm, Hom giống, IAA, IBA, $\alpha-N A A$.

\section{Tóm tắt}

Râu hùm (Tacca chantrieri Andre) là loài cây thuốc quý, có công dụng chữa bệnh thấp khớp, dùng uống trị viêm loét dạ dày và hành tá tràng, viêm gan, huyết áp cao, bỏng lửa, lở ngứa...[7,10]. Mặc dù chưa bị khai thác nhiều quá mức song nạn phá rừng và khai thác rừng đã trực tiếp làm thu hẹp diện phân bố và khả năng trữ lượng tự nhiên của cây. Việc nghiên cứu nhân giống loài Râu hùm nhằm bảo tồn và phát triển các loài cây thuốc của tỉnh Tuyên Quang. Kết quả nghiên cứu nhân giống Râu hùm bằng phương pháp sinh dưỡng cho thấy, hom giâm ở các vị trí hom khác nhau (hom ngọn, hom giữa và hom gốc) có kích thước $10 \mathrm{~cm}$ cho tỷ lệ sống cao hơn, trong đó hom giữa (kích thước $10 \mathrm{~cm}$ ) sau 30 ngày, 60 ngày và 90 ngày đều cho kết quả cao nhất với các giá trị tương ứng về tỷ lệ hom sống $(95,9 \%, 78,5 \%$ và $67,8 \%)$. Hom giữa $(10 \mathrm{~cm})$ cho số chồi/hom cao nhất. Khi nhân giống Râu hùm bằng hom giữa $(10 \mathrm{~cm})$ có sử dụng các chất kích thích sinh trưởng và nồng độ sử dụng các chất đó tới tỷ lệ sống và ra rễ cho thấy: sau 90 ngày, công thức cho tỷ lệ sống cao nhất là hom xử lý IAA với nồng độ 1500ppm đạt $100 \%$, cao hơn nhiều so với đối chứng. Tiếp đến là IBA với tỷ lệ $98,5 \%$, còn lại $\alpha$-NAA có sự tác động lên hom giâm thấp nhất. Loại chất kích thích sinh trưởng và nồng độ sử dụng đã ảnh hưởng rõ rệt đến tỷ lệ ra rễ và số rễ trung bình/hom, khi nồng độ tăng từ 500ppm lên đến 1000 ppm, đặc biệt lên đến $1500 p p m$ thì tỷ lệ ra rễ và số rễ trung bình/hom có $\mathrm{xu}$ hướng tăng theo. Công thức 9 với chất kích thích IBA ở nồng độ 1500 ppm cho tỷ lệ cao nhất.

\section{MỞ ĐÀ̀U}

Râu hùm (Tacca chantrieri Andre) là loại cỏ nhiều năm, thường gặp Râu hùm mọc ở ven suối, dưới tán rừng. Râu hùm phân bố nhiều ở các tỉnh Lào Cai, Yên Bái, Lạng Sơn, Tuyên Quang, Bắc Giang, Vĩnh Phúc, Hà Nội (Ba Vì), Hòa Bình, Ninh Bình, Nghệ an, Quảng Trị, Quảng Nam, Khánh Hòa, Kon Tum, Gia Lai, Lâm Đồng, Đắk Lắk, Đồng Nai [7,10].
Râu hùm là một trong những vị thuốc được dùng nhiều trong y học cổ truyền, thân rễ dùng ngoài chữa thấp khớp. Ở Trung Quốc, cây được dùng uống trị viêm loét dạ dày và hành tá tràng, viêm gan, huyết áp cao, đau dạ dày, bỏng lửa, lở ngứa [7,10].

Ở Na Hang (Tuyên Quang), Râu hùm được dân tộc Tày gọi là: $B o$ thác lủa; dân tộc Dao gọi là: Mào xam 
đòi. Dùng toàn cây làm thuốc. Dân tộc Tày dùng rễ phơi khô sắc nước uống chữa khớp; cả cây sắc uống chữa cao huyết áp; dân tộc Dao dùng rễ phơi khô đun nước uống chữa dạ dày, viêm phổi [7].

Mặc dù chưa bị khai thác nhiều quá mức, song nạn phá rừng và khai thác rừng đã trực tiếp làm thu hẹp diện phân bố và khả năng trữ lượng tự nhiên của cây. Việc nghiên cứu nhân giống nhằm bảo tồn và phát triển loài Râu hùm là rất cần thiết, có ý nghĩa khoa học và giá trị thực tiễn. Bài báo này trình bày kết quả nghiên cứu nhân giống sinh dưỡng (bằng hom) của Râu hùm được thực hiện tại trường Đại học Tân Trào, tỉnh Tuyên Quang.

\section{VẠT LIỆU VÀ PHƯƠNG PHÁP NGHIÊN CÚU}

\subsection{Vật liệu và địa điểm nghiên cứu}

- Hom giống Râu hùm được lấy từ các cây phân bố tự nhiên tại khu Bảo tồn thiên nhiên Na Hang. Việc nhân giống được thực hiện từ tháng 03 đến tháng 10 năm 2019 tại trường Đại học Tân Trào.

- Cát sạch, bình bơm thuốc sâu, giấy nilon trắng, dung dịchViben $\mathrm{C} 0,03 \%$, chất điều hòa sinh trưởng (IAA, IBA và $\alpha$-NAA).

- Túi bầu polyetylen kích thước $8 \times 12 \mathrm{~cm}$; hỗn hợp ruột bầu gồm $90 \%$ tầng đất mặt dưới tán rừng.

\subsection{Phương pháp nghiên cứu}

Nhân giống sinh dưỡng được thực hiện bằng cách giâm hom cắt từ thân rễ (củ). Việc giâm hom được thực hiện ngay sau khi thu từ cây mọc tự nhiên. Hom giâm được cắt bỏ phần lá phía gốc, sau đó được xử lý chống nấm, mốc; xử lý các chất điều hòa sinh trưởng và đưa vào các luống giâm.

Các thí nghiệm bao gồm:

Thí nghiệm 1: Ảnh hưởng của loại hom và kích thước hom đến tỷ lệ sống và tỷ lệ nảy chồi của hom giống. Loại hom trong thí nghiệm này bao gồm: hom ngọn, hom giữa (bánh tẻ) và hom gốc với các kích thước $5 \mathrm{~cm}, 10 \mathrm{~cm}$.

Thí nghiệm 2: Ảnh hưởng của loại chất điều hòa sinh trưởng và nồng độ sử dụng của các chất đó tới tỷ lệ sống và ra rễ của hom giâm.

Các chất điều hoà sinh trưởng dùng trong các thí nghiệm là: $\alpha$-NAA (Acid $\alpha$-napthilen acetic), IBA (Indol butyric acid), IAA (Indole Acetic Acid); mỗi chất được chia theo 3 loại nồng độ: $500 \mathrm{ppm}$; $1.000 \mathrm{ppm}$ và $1.500 \mathrm{ppm}$. Lô đối chứng không sử dụng chất điều hoà sinh trưởng.
Thí nghiệm gồm 10 công thức:

+ CT1: Xử lý các loại hom giống từ củ bằng IAA nồng độ 500ppm

+ CT2: Xử lý các loại hom giống từ củ bằng IAA nồng độ $1.000 \mathrm{ppm}$

+ CT3: Xử lý các loại hom giống từ củ bằng IAA nồng độ $1.500 \mathrm{ppm}$

+ CT4: Xử lý các loại hom giống từ củ bằng $\alpha$ NAA nồng độ 500ppm

+ CT5: Xử lý các loại hom giống từ củ bằng $\alpha$ NAA nồng độ 1.000ppm

+ CT6: Xử lý các loại hom giống từ củ bằng $\alpha$ NAA nồng độ $1.500 p p m$

+ CT7: Xử lý các loại hom giống từ củ bằng IBA nồng độ 500ppm

+ CT8: Xử lý bằng các loại hom giống từ củ IBA nồng độ $1.000 \mathrm{ppm}$

+ CT 9: Xử lý bằng các loại hom giống từ củ IBA nồng độ $1.500 \mathrm{ppm}$

+ CT10: Không xử lý hoá chất (Đối chứng)

Đối với các công thức xử lý các hom giống bằng IAA, $\alpha$-NAA và IBA, nhúng hom giâm vào dung dịch $1-2 \mathrm{~cm}$, thời gian xử lý hom kéo dài $20-30$ giây. Sau đó nhấc hom lên để ngoài không khí cho khô ráo mặt cắt trước khi ngâm, các thí nghiệm được lặp lại 3 lần. Sau khi giâm xong, hàng ngày tưới nước dạng phun sương 2 lần (sáng và chiều). Mỗi công thức sử dụng 90 hom. Các thí nghiệm được lặp lại 3 lần (tại 3 vườn khác nhau). Thời gian ghi số liệu sau 30,60 và 90 ngày. Mỗi lần ghi số liệu nhấc 30 hom/công thức/lần lặp để đo đếm các chỉ tiêu theo dõi.

\subsection{Phương pháp thu thập số liệu}

Trong thời gian thí nghiệm các công thức giâm hom số liệu được theo dõi hàng ngày và đo đếm các chỉ tiêu sau: Số hom sống, hom có chồi - số chồi; số hom ra rễ...

Nghiên cứu sự ảnh hưởng của các loại hom giống đến tỷ lệ sống, khả năng nảy chồi và ra rễ. Lựa chọn 3 loại hom từ củ: hom ngọn, hom giữa (bánh tẻ), hom già.

\subsection{Phương pháp xử lý số liệu}

Xử lý số liệu theo thống kê sinh học trong lâm nghiệp trên phần mềm Excel và SPSS theo Nguyễn Hải Tuất và cộng sự, 2005 [6]. 


\section{KÊTT QUẢ NGHIÊN CỨU}

3.1. Ảnh hưởng của loại hom và kích thước hom giâm đến khả năng ra rễ của hom giâm loài Râu hùm (Tacca chantrieri Andre)
Hom giống được thí nghiệm với 03 loại: hom ngọn, hom giữa (bánh tẻ) và hom gốc với các kích thước $5 \mathrm{~cm}, 10 \mathrm{~cm}$, được tiến hành vào mùa Xuân (tháng 3 năm 2019). Các kết quả thu được thể hiện trong bảng 1 .

Bảng 1: Ảnh hưởng của loại hom và kích thuơơ hom giống đến tỷ lệ sống của loài Râu hùm (Tacca chantrieri Andre) sau 90 ngày

\begin{tabular}{|l|r|r|r|r|r|r|}
\hline \multirow{2}{*}{} & \multicolumn{2}{|c|}{ Sau 30 ngày } & \multicolumn{2}{c|}{ Sau 60 ngày } & \multicolumn{2}{c|}{ Sau 90 ngày } \\
\cline { 2 - 7 } & Số hom sống & $\begin{array}{c}\text { Tỷ lệ } \\
(\%)\end{array}$ & Số hom sống & $\begin{array}{c}\text { Tỷ lệ } \\
(\%)\end{array}$ & Số hom sống & $\begin{array}{c}\text { Tỷ lệ } \\
(\%)\end{array}$ \\
\hline Hom ngọn $(5 \mathrm{~cm})$ & $83,3 \pm 0,6$ & 92,6 & $56,0 \pm 1,0$ & 62,2 & $26,0 \pm 1,0$ & 28,9 \\
\hline Hom ngọn (10cm) & $86,0 \pm 2,0$ & 95,6 & $63,7 \pm 0,6$ & 70,7 & $31,7 \pm 1,7$ & 34,4 \\
\hline Hom giữa $(5 \mathrm{~cm})$ & $85,3 \pm 1,2$ & 94,8 & $64,0 \pm 2,6$ & 71,1 & $49,7 \pm 2,5$ & 55,2 \\
\hline Hom giữa (10cm) & $\mathbf{8 6 , 3} \pm \mathbf{0 , 6}$ & $\mathbf{9 5 , 9}$ & $\mathbf{7 0 , 7} \pm \mathbf{0 , 6}$ & $\mathbf{7 8 , 5}$ & $\mathbf{6 1 , 0} \pm \mathbf{1 , 0}$ & $\mathbf{6 7 , 8}$ \\
\hline Hom gốc $(5 \mathrm{~cm})$ & $84,0 \pm 1,0$ & 93,3 & $44,7 \pm 0,6$ & 49,6 & $19,0 \pm 1,7$ & 21,1 \\
\hline Hom gốc $(10 \mathrm{~cm})$ & $85,7 \pm 2,5$ & 95,2 & $54,7 \pm 1,5$ & 60,7 & $24,0 \pm 2,6$ & 26,7 \\
\hline
\end{tabular}

Kết quả nghiên cứu thu được ở bảng 1 cho thấy, loại hom và kích thước hom có ảnh hưởng lớn đến khả năng nhân giống loài Râu hùm. Sau giâm 90 ngày, hom giữa, dài $10 \mathrm{~cm}$ có tỷ lệ sống cao nhất với $67,8 \%$; tiếp đến là hai công thức hom giữa $5 \mathrm{~cm}$ và hom ngọn $10 \mathrm{~cm}$ có tỷ lệ sống lần lượt là $55,2 \%$ và $34,4 \%$.

Xét theo loại hom thì ở cả hai công thức $(5 \mathrm{~cm}$ và $10 \mathrm{~cm}$ ) hom gốc đều cho tỷ lệ sống sau giâm 90 ngày thấp nhất. Điều này là do các hom gốc bị hóa gỗ, bề mặt lớp vỏ cũng hình thành các lớp bần mỏng nên quá trình biệt hóa, hình thành mô sẹo và rễ gặp nhiều khó

khăn hơn, diễn ra lâu hơn các loại hom khác dẫn đến tỷ lệ chết cao.

Xét về kích thước hom, tỷ lệ hom sống tăng dần theo kích thước hom trong cả ba loại hom, các hom có tỷ lệ sống cao nhất ở các công thức tương ứng với chiều dài $10 \mathrm{~cm}$.

Từ kết quả nhân giống ở trên, tác giả tiếp tục theo dõi ảnh hưởng của các loại hom giống có kích thước $10 \mathrm{~cm}$ đến tỷ lệ nảy chồi ở loài Râu hùm (Tacca chantrieri Andre) sau 30 ngày, 60 ngày, 90 ngày (bảng 2).

Bảng 2: Anh hưởng của các loại hom giống với kích thước 10 cm đến tỷ lệ nảy chồi ở loài Râu hùm (Tacca chantrieri Andre)

\begin{tabular}{|c|c|c|c|c|c|c|c|c|c|c|}
\hline \multicolumn{2}{|c|}{$\begin{array}{c}\text { Thời gian } \\
\text { Chỉ tiêu đánh giá }\end{array}$} & \multicolumn{3}{|c|}{ Sau 30 ngày } & \multicolumn{3}{|c|}{ Sau 60 ngày } & \multicolumn{3}{|c|}{ Sau 90 ngày } \\
\hline Chỉ tiêu đá & h giá & $\begin{array}{l}\text { Hom } \\
\text { ngọn } \\
10 \mathrm{~cm}\end{array}$ & $\begin{array}{c}\text { Hom } \\
\text { giữa } 10 \\
\mathrm{~cm}\end{array}$ & $\begin{array}{l}\text { Hom } \\
\text { gốc } 10 \\
\mathrm{~cm}\end{array}$ & $\begin{array}{l}\text { Hom } \\
\text { ngọn } \\
10 \mathrm{~cm}\end{array}$ & $\begin{array}{c}\text { Hom } \\
\text { giữa } 10 \\
\mathrm{~cm}\end{array}$ & $\begin{array}{l}\text { Hom } \\
\text { gốc } 10 \\
\mathrm{~cm}\end{array}$ & $\begin{array}{l}\text { Hom } \\
\text { ngọn } \\
10 \mathrm{~cm}\end{array}$ & $\begin{array}{c}\text { Hom } \\
\text { giữa } 10 \\
\mathrm{~cm}\end{array}$ & $\begin{array}{l}\text { Hom } \\
\text { gốc } 10 \\
\mathrm{~cm}\end{array}$ \\
\hline \multicolumn{2}{|c|}{$\begin{array}{l}\text { Số cây sống (tỷ lệ } \\
\% \text { ) }\end{array}$} & $\begin{array}{c}86,0 \pm \\
2,0 \\
(95,6 \%)\end{array}$ & $\begin{array}{c}86,3 \pm \\
0,6 \\
(95,9 \%)\end{array}$ & $\begin{array}{c}85,7 \pm \\
2,5 \\
(95,2 \%)\end{array}$ & $\begin{array}{c}63,7 \pm \\
0,6 \\
(70,7 \%)\end{array}$ & $\begin{array}{c}70,7 \pm \\
0,6 \\
(78,5 \%)\end{array}$ & $\begin{array}{c}54,7 \pm \\
1,5 \\
(60,7 \%)\end{array}$ & $\begin{array}{c}31,7 \pm \\
1,7 \\
(34,4 \%)\end{array}$ & $\begin{array}{c}61,0 \pm \\
1,0 \\
(67,8 \%)\end{array}$ & $\begin{array}{c}24,0 \pm \\
2,6 \\
(26,7 \%)\end{array}$ \\
\hline \multirow{3}{*}{$\begin{array}{l}\text { Số } \\
\text { chồi/Hom }\end{array}$} & 1 chồi & 86 & 45 & 33 & 63 & 54 & 21 & 31 & 41 & 19 \\
\hline & 2 chồi & - & 2 & - & - & 7 & 3 & - & 16 & 5 \\
\hline & $\begin{array}{l}>2 \\
\text { chồi }\end{array}$ & - & - & & - & - & - & - & 4 & - \\
\hline
\end{tabular}


Qua bảng 2 cho thấy, sau giâm 30 và 60 ngày hom ngọn vì có mô phân sinh ngọn nên số chồi/hom đạt tỷ lệ cao nhất là $86 / 90$ và $63 / 90$, nhưng sau do tỷ lệ sống giảm nên số chồi/hom lại thấp hơn so với hom giữa.

Sau giâm 90 ngày hom giữa số chồi/hom đạt cao nhất, các cây còn sống đều nảy chồi, đặc biệt không chỉ có 1 chồi/hom mà từ 2 chồi trở lên/hom. Nếu tính số lượng chồi/hom, hom giữa đạt tỷ lệ cao nhất, sau đến hom gốc, cuối cùng là hom ngọn, hom giữa có 20 hom đạt từ 2 chồi/hom trở lên, so với hom gốc là 5 hom và hom ngọn không có hom đạt 2 chồi/hom.

3.2. Ảnh hưởng của loại chất kích thích sinh trưởng và nồng độ sử dụng của các chất đó tới tỷ lệ sống và ra rễ của hom giâm ở loài Râu hùm (Tacca chantrieri Andre)

Từ kết quả thử nghiệm trên đối với các kích thước của hom, đề tài đã sử dụng các chất kích thích sinh trưởng và nồng độ sử dụng tới hom giữa $(10 \mathrm{~cm})$ cho các thí nghiệm tiếp theo.

Ở kết quả bảng 3, sau giâm 30 ngày đầu tiên, về cơ bản tất cả các hom giâm đều còn sống nhưng sau giâm
60 ngày thì số lượng các hom sống đã giảm và có sự khác biệt giữa các công thức. Sau giâm 90 ngày, tất cả các công thức thí nghiệm đều cho tỷ lệ hom sống cao hơn lô đối chứng (không xử lý chất kích thích sinh trưởng) với các mức độ khác biệt không đồng đều. Nếu so sánh cùng nồng độ, thì tất cả các thí nghiệm xử lý bằng IAA đều cho hiệu quả cao hơn so với hai chất kích thích còn lại, tiếp đến là IBA và $\alpha-\mathrm{NAA}$ có sự tác động lên hom giâm thấp nhất. Công thức cho tỷ lệ sống cao nhất là hom xử lý IAA với nồng độ 1.500ppm đạt $100 \%$, cao hơn nhiều so với lô đối chứng.

Như vậy, từ kết quả trên cho ta thấy đối với nhân giống bằng hom ở loài Râu hùm, việc dùng các loại chất kích thích sinh trưởng với các nồng độ khác nhau cũng cho kết quả tỷ lệ sống khác nhau. Bước đầu nghiên cứu với loại kích thích sinh trưởng IAA nồng độ $1.500 p p m$, sau 90 ngày cho tỷ lệ hom sống cao nhất, tuy nhiên giá trị cao nhất nằm ở giá trị biên của thí nghiệm nên cần có thêm các thí nghiệm với nồng độ xử lý IAA lớn hơn hoặc bằng $1.500 p p m$ để tìm ra nồng độ tối ưu.

Bảng 3: Anh hưởng của các chất kích thích sinh trưởng

và nồng độ sử dụng đến tỷ lệ sống của hom giữa (10cm) ở loài Râu hùm (Tacca chantrieri Andre)

\begin{tabular}{|c|c|c|c|c|c|c|c|c|}
\hline \multirow{2}{*}{$\begin{array}{c}\text { Công } \\
\text { thức }\end{array}$} & \multirow{2}{*}{$\begin{array}{c}\text { Hóa } \\
\text { chất }\end{array}$} & \multirow{2}{*}{$\begin{array}{c}\text { Nồng độ } \\
(\mathbf{p p m})\end{array}$} & \multicolumn{2}{|c|}{$\mathbf{3 0}$ ngày } & \multicolumn{2}{|c|}{$\mathbf{6 0}$ ngày } & \multicolumn{2}{c|}{$\mathbf{9 0}$ ngày } \\
\cline { 5 - 9 } & & Sống & $\begin{array}{c}\text { Tỷ lệ } \\
(\%)\end{array}$ & Số hom sống & $\begin{array}{c}\text { Tỷ lệ } \\
(\%)\end{array}$ & $\begin{array}{c}\text { Số hom } \\
\text { sống }\end{array}$ & $\begin{array}{c}\text { Tỷ lệ } \\
(\%)\end{array}$ \\
\hline CT1 & IAA & 500 & $86,3 \pm 0,6$ & 95.9 & $81,7 \pm 1,5$ & 90,7 & $77,7 \pm 1,5$ & 86.3 \\
\hline CT2 & IAA & $\mathbf{1 . 0 0 0}$ & $\mathbf{8 9 , 0} \pm \mathbf{1 , 0}$ & $\mathbf{9 8 . 9}$ & $\mathbf{8 7 , 7} \pm \mathbf{0 , 6}$ & $\mathbf{9 7 , 4}$ & $\mathbf{8 7 , 0} \pm \mathbf{1 , 0}$ & $\mathbf{9 6 . 7}$ \\
\hline CT3 & IAA & $\mathbf{1 . 5 0 0}$ & $\mathbf{9 0 , 0} \pm \mathbf{0 , 0}$ & $\mathbf{1 0 0 . 0}$ & $\mathbf{9 0 , 0} \pm \mathbf{0 , 0}$ & $\mathbf{1 0 0 , 0}$ & $\mathbf{9 0 , 0} \pm \mathbf{0 , 0}$ & $\mathbf{1 0 0 . 0}$ \\
\hline CT4 & $\alpha$-NAA & 500 & $82,7 \pm 1,5$ & 91.9 & $73,3 \pm 0,6$ & 81,5 & $70,0 \pm 1,0$ & 77.8 \\
\hline CT5 & $\alpha$-NAA & 1.000 & $88,3 \pm 0,6$ & 98.1 & $83,7 \pm 0,6$ & 93,0 & $81,0 \pm 1,0$ & 90.0 \\
\hline CT6 & $\mathbf{\alpha - N A A}$ & $\mathbf{1 . 5 0 0}$ & $\mathbf{9 0 , 0} \pm \mathbf{0 , 0}$ & $\mathbf{1 0 0 . 0}$ & $\mathbf{8 8 , 7} \pm \mathbf{0 , 6}$ & $\mathbf{9 8 , 5}$ & $\mathbf{8 6 , 3} \pm \mathbf{0 , 6}$ & $\mathbf{9 5 . 9}$ \\
\hline CT7 & IBA & 500 & $83,3 \pm 1,2$ & 92.6 & $74,7 \pm 1,2$ & 83,0 & $73,0 \pm 1,0$ & 81.1 \\
\hline CT8 & IBA & 1.000 & $85,7 \pm 0,6$ & 95.2 & $84,7 \pm 0,6$ & 94,1 & $83,3 \pm 0,6$ & 92.6 \\
\hline CT9 & IBA & $\mathbf{1 . 5 0 0}$ & $\mathbf{9 0 , 0} \pm \mathbf{0 , 0}$ & $\mathbf{1 0 0 . 0}$ & $\mathbf{8 9 , 3} \pm \mathbf{0 , 6}$ & $\mathbf{9 9 , 3}$ & $\mathbf{8 8 , 7} \pm \mathbf{1 , 5}$ & $\mathbf{9 8 . 5}$ \\
\hline CT10 & ĐC & & $82,7 \pm 2,5$ & 91.9 & $63,3 \pm 1,5$ & 70,4 & $54,0 \pm 1,0$ & 60.0 \\
\hline
\end{tabular}

Từ kết quả bảng 4 và bảng 5 ở trên ta thấy, sự khác biệt về số rễ trung bình/hom giữa công thức CT9 (IBA $1.500 p p m)$ với các công thức thí nghiệm khác. Sau giâm 90 ngày tất cả các hom sống đều ra rễ.

Sau thời gian 90 ngày công thức đối chứng có tỷ lệ số hom ra rễ thấp nhất với $60 \%$, số rễ trung bình trên hom cũng đạt thấp nhất là 3,33 rễ/hom, chiều dài trung bình đạt $1,13 \mathrm{~cm}$. Ở công thức 9 có số hom ra rễ cao nhất với $98,5 \%$, số rễ trung bình trên hom cao nhất là 7,67 hom và chiều dài rễ trung bình là $3,17 \mathrm{~cm}$.

Như vậy, loại chất kích thích sinh trưởng và nồng độ sử dụng khác nhau đã ảnh hưởng rõ rệt đến tỷ lệ ra rễ và số rễ trung bình/hom, khi nồng độ tăng từ $500 \mathrm{ppm}$ lên đến $1.000 \mathrm{ppm}$, đặc biệt lên đến $1.500 \mathrm{ppm}$ thì tỷ lệ ra rễ và số rễ trung bình/hom có xu hướng tăng theo. Công thức 9 với chất kích thích IBA ở nồng độ 1.500ppm cho tỷ lệ cao nhất. Chứng tỏ công thức 9 ảnh hưởng trội nhất đến khả năng ra rễ và số rễ cũng như chiều dài rễ trên hom của loài Râu hùm. 
Bảng 4: Ảnh hưởng của các chất kích thích sinh trương và nồng độ sủ dụng đến tỷ lệ ra rễ của hom giữa (10cm) ở loài Râu hùm (Tacca chantrieri Andre)

\begin{tabular}{|c|c|c|c|c|c|c|c|c|}
\hline \multirow[b]{2}{*}{$\begin{array}{l}\text { Công } \\
\text { thức }\end{array}$} & \multirow[b]{2}{*}{ Hóa chất } & \multirow[b]{2}{*}{$\begin{array}{c}\text { Nồng } \\
\text { độ } \\
\text { (ppm) }\end{array}$} & \multicolumn{2}{|c|}{30 ngày } & \multicolumn{2}{|c|}{60 ngày } & \multicolumn{2}{|c|}{90 ngày } \\
\hline & & & Số hom ra rễ & $\begin{array}{c}\text { Tỷ lệ } \\
(\%)\end{array}$ & $\begin{array}{c}\text { Số hom ra } \\
\text { rễ }\end{array}$ & $\begin{array}{l}\text { Tỷ lệ } \\
(\%)\end{array}$ & $\begin{array}{l}\text { Số hom } \\
\text { ra rễ }\end{array}$ & Tỷ lệ (\%) \\
\hline CT1 & IAA & 500 & $16,7 \pm 0,6$ & 18,5 & $35,7 \pm 1,5$ & 39,6 & $77,3 \pm 0,6$ & 85,9 \\
\hline CT2 & IAA & 1.000 & $28,7 \pm 0,6$ & 31,9 & $53,3 \pm 1,2$ & 59,3 & $87,0 \pm 1,0$ & 96.7 \\
\hline CT3 & IAA & 1.500 & $44,3 \pm 0,6$ & 49,3 & $61,7 \pm 0,6$ & 68,5 & $90,0 \pm 0,0$ & 100.0 \\
\hline CT4 & $\alpha$-NAA & 500 & $12,0 \pm 1,0$ & 13,3 & $29,0 \pm 1,0$ & 32,2 & $70,0 \pm 1,0$ & 77.8 \\
\hline CT5 & $\alpha$-NAA & 1.000 & $24,3 \pm 1,2$ & 27,0 & $45,3 \pm 2,3$ & 50,4 & $81,0 \pm 1,0$ & 90.0 \\
\hline CT6 & $\alpha$-NAA & 1.500 & $34,7 \pm 0,6$ & 38,5 & $54,7 \pm 0,6$ & 60,7 & $86,3 \pm 0,6$ & 95.9 \\
\hline CT7 & IBA & 500 & $15,3 \pm 0,6$ & 17,0 & $34,0 \pm 2,0$ & 37,8 & $73,0 \pm 1,0$ & 81.1 \\
\hline CT8 & IBA & 1.000 & $28,3 \pm 0,6$ & 31,5 & $49,3 \pm 0,6$ & 54,8 & $83,3 \pm 0,6$ & 92.6 \\
\hline CT9 & IBA & 1.500 & $42,3 \pm 0,6$ & 47,0 & $59,3 \pm 0,6$ & 65,9 & $88,7 \pm 1,5$ & 98.5 \\
\hline CT10 & ĐC & & $10,3 \pm 0,6$ & 11,5 & $23,0 \pm 1,0$ & 25,6 & $54,0 \pm 1,0$ & 60.0 \\
\hline
\end{tabular}

Bảng 5: Ảnh hưởng của các loại chất kích thích sinh trưởng và nồng độ của chúng đến số rễ trung bình/hom và chiều dài rễ trung bình

\begin{tabular}{|c|c|r|r|r|r|c|}
\hline $\begin{array}{c}\text { Công } \\
\text { thức }\end{array}$ & Hóa chất & $\begin{array}{c}\text { Nồng độ } \\
\text { (ppm) }\end{array}$ & \multicolumn{1}{c|}{$\begin{array}{c}\text { Số hom } \\
\text { ra rễ }\end{array}$} & \multicolumn{1}{c|}{$\begin{array}{c}\text { Tỷ lệ } \\
(\mathbf{\%})\end{array}$} & $\begin{array}{c}\text { Số rễ trung } \\
\text { bình/hom }\end{array}$ & $\begin{array}{c}\text { Chiều dài rễ } \\
\text { trung bình } \\
(\mathbf{c m})\end{array}$ \\
\hline CT1 & IAA & 500 & $77,3 \pm 0,6$ & 85,9 & $4,67 \pm 0,58$ & $1,18 \pm 0,10$ \\
\hline CT2 & IAA & 1.000 & $87,0 \pm 1,0$ & 96.7 & $5,00 \pm 0,00$ & $1,51 \pm 0,10$ \\
\hline CT3 & IAA & 1.500 & $90,0 \pm 0,0$ & 100.0 & $5,67 \pm 0,58$ & $2,01 \pm 0,12$ \\
\hline CT4 & $\alpha$-NAA & 500 & $70,0 \pm 1,0$ & 77.8 & $5,00 \pm 0,00$ & $1,22 \pm 0,06$ \\
\hline CT5 & a-NAA & 1.000 & $81,0 \pm 1,0$ & 90.0 & $5,33 \pm 0,58$ & $2,13 \pm 0,12$ \\
\hline CT6 & a-NAA & 1.500 & $86,3 \pm 0,6$ & 95.9 & $5,67 \pm 0,58$ & $1,88 \pm 0,11$ \\
\hline CT7 & IBA & 500 & $73,0 \pm 1,0$ & 81.1 & $4,67 \pm 0,58$ & $1,86 \pm 0,13$ \\
\hline CT8 & IBA & 1.000 & $83,3 \pm 0,6$ & 92.6 & $5,67 \pm 0,58$ & $2,20 \pm 0,56$ \\
\hline CT9 & IBA & $\mathbf{1 . 5 0 0}$ & $\mathbf{8 8 , 7} \pm \mathbf{1 , 5}$ & $\mathbf{9 8 . 5}$ & $\mathbf{7 , 6 7 \pm 0 , 5 8}$ & $\mathbf{3 , 1 7 \pm 0 , 1 0}$ \\
\hline CT10 & ĐC & & $54,0 \pm 1,0$ & 60.0 & $3,33 \pm 0,58$ & $1,13 \pm 0,15$ \\
\hline
\end{tabular}

\section{KẾT LUẬN}

1. Râu hùm (Tacca chantrieri Andre) nhân giống sinh dưỡng được thực hiện bằng cách giâm hom cắt từ thân rễ (củ) có kích thước $10 \mathrm{~cm}$ cho tỷ lệ sống cao hơn, trong đó hom giữa $(10 \mathrm{~cm})$ sau giâm 90 ngày cho kết quả cao hơn so với hom ngọn và hom gốc với các giá trị tương ứng về tỷ lệ hom sống là $(95,9 \%, 78,5 \%$ và $67,8 \%)$.

2. Sử dụng hom giữa với kích thước hom giâm $10 \mathrm{~cm}$ có tỷ lệ số hom lớn hơn 2 chồi/hom đạt cao nhất, sau đến hom gốc, cuối cùng là hom ngọn. Sau giâm 90 ngày, hom giữa có 20 hom đạt từ 2 chồi/hom trở lên, so với hom gốc là 5 hom và hom ngọn không có hom đạt 2 chồi/hom.

3. Sau 90 ngày nhân giống Râu hùm bằng hom giữa $(10 \mathrm{~cm})$ có sử dụng các chất kích thích sinh trưởng, hom xử lý IAA với nồng độ $1.500 p p m$ cho tỷ lệ sống cao nhất, đạt $100 \%$, tiếp đến là IBA với tỷ lệ 98,5\%, còn lại $\alpha$-NAA có sự tác động lên hom giâm thấp nhất. Công thức xử lý IBA ở nồng độ $1.500 p p m$ cho tỷ lệ số hom ra rễ và số rễ trung bình/hom đạt cao nhất. 


\section{TÀI LIỆU THAM KHẢO}

1. Do Huy Bich, Dang Quang Chung, Bui Xuan Chuong, Nguyen Thuong Dong, Do Trung Dam, Pham Van Hien, Vu Ngoc Lo, Pham Duy Mai, Pham Kim Man, Doan Thi Nhu, Nguyen Tap, Tran Toan (2006). Medicinal plants and medicinal animals in Vietnam, vol. 1, pages 940-943. Publisher: Science and Technology, Hanoi.

2. Do Tat Loi (2006), Vietnamese medicinal plants and herbs, Hanoi Medical Publisher, Hanoi.

3. Le Dinh Kha, Nguyen Hoang Nghia, Pham Van Tuan, Doan Thi Bich (1997), Research on cuttings of Eucalyptus. Results of scientific research on forest seed selection, T2. Agricultural Publisher: p. 84-94.

4. Le Dinh Kha et al (2003), Selection, creation and propagation of some major forest plant species in Vietnam, Agriculture Publisher:Hanoi.

5. Nguyen Mong Hung (2005), Plant breeding techniques, Agriculture Publisher Hanoi.
6. Nguyen Hai Tuat, Nguyen Trong Binh (2005), Exploiting and using SPSS for data processing in Forestry, Agriculture Publisher Hanoi.

7. Nguyen Thi Hai (2018), research medicinal plant resources to propose solutions to conserve and sustainably use a number of valuable species in $\mathrm{Na}$ Hang Nature Reserve, Tuyen Quang province, PhD thesis in biology, Hanoi.

8. Nguyen Tien Ban (Editor, 2003), List of Plant Species of Vietnam, vol. 2, Agriculture Publisher, Hanoi, pp. 144-145.

9. Institute of Medicinal Materials (2016), List of Vietnamese medicinal plants, Science and Technology Publisher, Hanoi, page 437.

10. Vo Van Chi, Dictionary of Vietnamese medicinal plants. Vol. 1, 2, Hanoi Medical Publisher, Hanoi,2012.

\section{Propagation of species of taciturne (Tacca chantrieri Andre) by vegetative propagation at Tan Trao University, Tuyen Quang}

Nguyen Thi Hai, Tran Thi Thanh Van, Chu Quynh Mai

\section{Article info}

Recieved:

20/4/2020

Accepted:

$12 / 8 / 2020$

Keywords:

Cuttings, Antennae,

Breeding Hom, IAA,

$I B A, \alpha-N A A$.

\begin{abstract}
Tacca chantrieri Andre (Tacca chantrieri Andre) is a valuable medicinal plant, used to treat rheumatism, stomach and duodenal ulcers, hepatitis, high blood pressure, burning, itchy sores ... [7,10 ]. Despite not being overexploited, deforestation and forest exploitation have directly narrowed the distribution area and the capacity of the tree's natural reserves. Research on propagating lobster species is to conserve and develop medical plants in Tuyen Quang province Results of research on propagating lobster by vegetative method showed that cuttings at different places (top cuttings, middle cuttings and root cuttings) which had a size of $10 \mathrm{~cm}$ for a higher survival rate, in which middle cuttings $(10 \mathrm{~cm}$ size) after 30 days, 60 days and 90 days all showed the highest results with survival rate $(95.9 \%, 78.5 \%$ and $67.8 \%)$. Middle cuttings $(10 \mathrm{~cm})$ gave the highest number of shoots / cuttings. When propagating the Tacca chantrieri Andre with middle cuttings $(10 \mathrm{~cm})$ using growth stimulants and the concentration of those substances used for survival and rooting showed that: after 90 days, the formula for the highest survival rate is a curing IAA with a concentration of $1500 \mathrm{ppm}$ reaching $100 \%$, much higher than the control. IBA was followed by $98.5 \%$, the remaining $\alpha$-NAA had the lowest impact on cuttings. The type of growth stimulant and the concentration have markedly affected the rooting rate and the average number of roots / cuttings, when the concentration increased from 500ppm to $1000 \mathrm{ppm}$, especially up to $1500 \mathrm{ppm}$, the rooting rate and the number of average roots / cutted tended to increase. Formula 9 with stimulant IBA at the concentration of $1500 \mathrm{ppm}$ gives the highest rate.
\end{abstract}

